\title{
Almighty, the Universe
}

\section{IMH Muhammad Akil}

Provincial Government Service, Haryana, Chandigarh, India

\section{Email address:}

mohd.akil68@yahoo.com

\section{To cite this article:}

IMH Muhammad Akil. Almighty, the Universe. American Journal of Physics and Applications. Vol. 1, No. 3, 2013, pp. 99-103. doi: 10.11648/j.ajpa.20130103.17

\begin{abstract}
The beginning and end; size, shape and centre of the Universe; who created it are some of the most basic riddles pertaining to the Universe which are still unanswered. Also, the quest for most basic particle which is the basis of the Universe is still unquenched. The author has researched these riddles in detail and has postulated seven laws governing the functioning of the Universe. The laws are the Universe can neither be created nor destroyed; the Universe exists since infinite time and will exist till infinite time; the Universe has an infinite size; the Universe is in a state of dynamic equilibrium; all the activities in the Universe are uncertain but within certain certainty limits; every constituent of the Universe is unique; every constituent of the Universe has a unique dimension known as life dimension. There could be innumerable number of visible and invisible species living in various parts of the Universe, all of them passing through the cyclical life spans and doing their bit for maintaining the dynamic equilibrium. Constituents with temporarily shorter life spans appear alive to us while those with relatively longer ones appear inert, though all have life and are made up of live smaller constituents. The Universe is continuously changing, rearranging and evolving itself. Depending upon the relative intensity of the physical, non-physical and the need forces the Universe continuously rearranges itself so that a dynamic equilibrium is always maintained. In short, whatever is needed by a constituent for the most efficient functioning of the Universe is being provided by the Universe to it at the most suitable point of time. It has been concluded that the Universe is God and the God is Universe. Both are exactly one and the same. Only the names are different. It is the entirety of the Universe including the mass, energy, forces and the collective life in Its constituents which we perceive as the God Almighty. He is indivisible, uncuttable, un-replicable, un-replaceable, un-create able, un-destroyable and unique. He is only one, whom we address by different names in different parts of our very small planet. He is the Creator of Himself embodying the life in the collective form who by Himself sitting on the driver's seat manages His affairs in the best possible and the most efficient manner.
\end{abstract}

Keywords: Almighty, the Universe, the Universal Riddles, the Universal Laws of the Universe, Imhon, Lubon, Priyon, Unon

\section{Introduction}

The best minds in this world are of the opinion that the life exists on the planet earth, approximately, for the last some billion years. The figure appears to be purely guesswork. It could be any other figure, say in billion years, or in trillion years as well. We are even more ignorant about the number of stars in our galaxy or the number of galaxies in the Universe. The oft-quoted figures run into billions and trillions.

Similarly, a lot of effort has gone in figuring out the centre of the Universe, Its age and shape, Its beginning and end etc. Then, some scientists have worked extremely hard to discover the elementary basic particle which has been used in the formation of various constituents of the Universe. But the results achieved by them have been and are being revised or altered by their successors after very short periods of time.

Extensive theoretical work is being conducted to decide whether the Universe is expanding, contracting or is static. Big Bang theory has been propagated to explain the beginning of the Universe assuming It to begin at some definite point of time. Theory of general relativity tried to explain some of the unanswered questions, but very soon, a more refined theory known as Superstring theory had to be developed to explain some conflicting results arising out of the relativity theory.

The discovery of time dimension was an important milestone in the man's quest for knowledge. However, the direction of time, as also whether it is reversible or not is still 
puzzling us. Similarly, discovery of carcasses of stars, popularly known as the black holes, in the Universe made the scientific world ecstatic with joy and now it is guessed that a black hole would disappear completely in a tremendous final burst of energy emission. We shall realize that this much knowledge could percolate down to us only after a large number of thinkers, religious leaders, physicists, astronomers, mathematicians and other naturally gifted luminaries have made significant contributions towards the behaviour of the great Universe. Aristotle, Ptolemy, Copernicus, Newton, Galileo, Darwin, Einstein, Heisenberg, Stephen Hawking, Brian Greene etc are some of the well-known personalities who have delved deep into the vast mysteries of the Universe to enlighten the mankind on Its majesty. The list is unending but it is difficult to mention the names of all known names in this field. However, it will certainly not belittle the efforts of those luminaries who either could not or did not express their understanding of the issue; or their work could not get its due recognition due to some unknown and inexplicable reasons.

Aristotle argued that the earth was spherical in shape instead of a flat surface as was considered earlier. Ptolemy, in his Almagest, presented a geo-centric model of the Universe. While Copernicus thought that the sun was stationary and the planets moved in circular orbits around it. But later on, Newton's law of universal gravitational attraction proved that the celestial bodies follow elliptical orbits instead of the circular ones.

At around the same time, Charles Darwin, the celebrated naturalist mesmerized the mankind by propagating the evolutionary theory and the process of natural selection in species.

Researches by Stephen Hawking, the supernova of world physics on black holes and by Brian Greene on superstring theory have taken us somewhat nearer to the minimum satisfaction level.

However, despite the tremendous efforts made by various luminaries during the past thousands of years, the Universe has still remained a big, large and complicated riddle eluding permanently satisfactory replies to some of the most natural queries which regularly take birth inside the human minds.

\subsection{Aim}

Our aim will be to find truthful replies, in simple language, to the most basic queries pertaining to the Universe. Age old knowledge regarding hitherto scientifically undefined forces will be crystallized. A model comprising of seven laws governing the Universe will be developed and presented before the mankind.

Firstly, the universal riddles will be identified followed by the presentation of the Universal laws governing the functioning of the Universe. Lastly, in the conclusion part, the Creator of the Universe will be discussed.

\subsection{Methodology}

Let us list these most natural queries; the replies achieved till date; discuss their correctness and adequacy; try to figure out the scope for further improvement; and develop a model which is capable of truthfully and adequately solving the riddles taking birth in the simplest of human minds. The test of the model shall be in quenching the intellectual thirst of anybody who looks at and feel the great Universe with respect, compassion and pride.

\section{The Universal Riddles}

\subsection{Beginning and End of the Universe? Direction of Time Dimension?}

Some scientists believe that the Universe came into existence at a time when an event called the Big Bang occurred. At that point of time, the Universe was supposedly infinitesimally small and, therefore, infinitely dense. But the main point again remains unanswered as to when this infinitely dense and infinitesimally small Universe came into existence. The Big Bang theory appears to be a result of observing some expansion in the Universe. The observation appears to be correct at the present moment but its extrapolation that it is only expanding and expanding since times immemorial may not be correct. It may be cyclically expanding and contracting as It is dynamic having life in It and certainly not static. There is no meeting of minds amongst the scientists regarding Its origin and end. In the words of Stephen Hawking '... but if the universe is really self contained, having no boundary or edge, it would be neither created nor destroyed. It would simply be. What place, then, for a creator?...'

We will try to effectively remove this ambiguity as to the role of the creator.

The direction and reversibility of time dimension troubles us when we start manufacturing theories based on very limited observations that too for an even more limited time-periods. The research and the subsequent findings till date have not been able to decide the direction and reversibility of time.

\subsection{Size and shape of the Universe?}

No unity could be developed amongst the scientists as to the size and shape of the Universe. They could only say that It is expanding at the moment. They could not even decide whether there could be a unified theory to explain the Universe's behaviour.

\subsection{Centre of the Universe?}

Some thinkers declared that a specific point on the earth is the centre while some scientists declared sun as the centre. Surprisingly, not much discussion or work could be undertaken to differentiate between the absolute and relative spatial dimensions. Resultantly, the guesswork is still continuing on the issue. Needless to say, that the whole issue of finding the centre of the Universe appears to be a purely academic exercise. 


\subsection{Why the Universe is Expanding?}

As a result of the observation of the expansion in the Universe, Big Bang theory was postulated. It was also observed that the entropy of the Universe is increasing at the moment. It was not thought that the most natural thing should be an increase in entropy for some time-period; then decrease in its rate of increase; a zero level in the rate and then a decrease in the entropy and so on. This change in entropy should follow the most natural pattern of a wave as is true with all other natural processes. The same wave pattern of expansion and contraction appears to being followed by the Universe as it will keep the Universe in equilibrium.

\subsection{What Happens Ultimately to a Black Hole?}

As already discussed, Stephan Hawking mentions in his book ' Theory of Everything' that a black hole would disappear completely in a tremendous final burst of energy emission. But why only this form of disappearance and what will happen to this emitted energy? Can't the particles of the emitted energy convert into mass particles, bond with the other like-minded particles and form an altogether new constituent of the Universe. The present theories are silent on the issue.

\subsection{What is the Most Basic Constituent Particle of the Universe?}

World over, the scientists are searching frantically the so called 'most basic particle' of the Universe. Initially, they discovered atom as the most basic particle, then came the electrons, protons and neutrons as the elementary particles. Then came quarks, up quarks, down quarks; charm quarks, strange quarks; top quarks, bottom quarks etc. Similarly, we have gluon, photon, boson and graviton as force particles respectively of strong, electromagnetic, weak and gravity forces. These force particles are also not final as they are further being modified. Discovery of Higgs' boson is one such example. The list is unending as we are regularly being bombarded by discoveries of new basic particles. And now a situation has reached when nobody seems to appear ready to seriously take notice of discovery claims of new fundamental or basic particles. Even after thousands of years after first such claim, the scientists are still eagerly awaiting the discovery of the true basic fundamental particle which forms the matter, the energy and the force which is uncuttable but repeatable.

\subsection{Who Created the Universe and Why? What is God, the Almighty?}

It is the most natural and the most thought question but very less asked for fear of being branded as unfaithful by certain self declared God men. The question ' Who created the Universe and Why?' has been attempted by many great thinkers with focus on some outside agency as the Creator. The outside agency concept created even more questions than providing solutions to the existing queries. When the Creator could not be found, the question 'Why the Universe was created' has also remained unanswered till date. Some scientists even argue whether there is any place in the Universe for a supernatural power for creation of the Universe, Its governance or Its destruction, if any. They also argue whether the existence of such a superpower is needed at all.

\section{Universal Laws of the Universe}

Now, we have reached a stage where it is possible for us to give an in-depth consideration to all the issues by looking from inside, in all possible directions and in all possible dimensions.

I may begin by listing the most natural laws governing the behaviour of the Universe. The word 'listing' has been used in this paragraph as the laws are already in existence. I shall only attempt to crystallize, segregate and write them in a language which could be understood by anybody and everybody. The following seven laws are listed:

\subsection{The Universe can neither be Created nor Destroyed}

It is as it is. Not even the smallest particle could be created or destroyed. A particle can only be transformed into other states, for example from mass to energy or vice-versa.

\subsection{The Universe Exists since Infinite Time and will Exist Till Infinite Time}

That is to say, the Universe is moving from minus infinity to plus infinity while always denoting the present moment as zero on this absolute scale of time. However, we can always measure the time with respect to an assumed or a given reference frame.

\subsection{The Universe has an Infinite Size}

All the spatial dimensions of the Universe are spread from minus infinity to plus infinity, making the absolute location of any point as zero. That is, every point in the Universe could be considered as the centre of the Universe. Looking at the hindsight, now we can say that Ptolemy was not entirely incorrect when he argued for the earth as the centre of the Universe. But any other point could also be the centre as well.

However, it is always possible to assign values to the spatial dimensions of any point in the Universe with respect to an assumed or a given frame of reference.

As Its dimensions measure from minus infinity to plus infinity and It is in a state of perpetual dynamism, no fixed shape could be possible for It. Neither there is any need for It to have a fixed shape. Therefore, the Universe has no fixed shape.

\subsection{The Universe is in a State of 'Dynamic Equilibrium'}

It is continuously changing and rearranging itself. Sometimes it appears to be expanding and at some other 
times it may appear to be contracting.

All the constituents of the Universe are also in a state of perpetual dynamism. Nothing is static in the Universe. Everything is continuously changing and rearranging itself, always trying to maintain equilibrium.

Every process in and of the Universe is in a cyclic or wave form with continuously changing wavelength and amplitude, but within certain limits.

\subsection{All the Activities in the Universe are Uncertain but within Certain 'Certainty Limits'}

Every process in and of the Universe is uncertain but the uncertainty is not without limits. On the contrary, it is always within some certainty limits. These certainty limits may vary with variable circumstances and time.

As every event is uncertain, it will not be possible for anybody or any machine to enter into future, observe the events there and come back and reveal it to others. However, based on certain observations, we may guess or extrapolate regarding the future events. But it should be considered purely as guesswork and nothing more.

\subsection{Every Constituent of the Universe is Unique}

Though on a macroscopic level the Universe constituents' behaviour appears almost similar to that of other constituents yet on a microscopic level every constituent is different from all other constituents of the Universe at all points of time. Also, every constituent is different at different points of time.

This leads us to the fact that no such thing like a "basic particle" of matter or force or energy exists in the Universe.

\subsection{Every Constituent of the Universe has a unique Dimension Known as 'Life Dimension' in addition to its Spatial and Time Dimensions}

Everything in the Universe ranging from the smallest particle to human-beings to plants to inert materials to planets, stars, galaxies etc are born, grow up, temporarily release some energy (expire), disintegrate into smaller constituents, get absorbed in the cosmos, recharge themselves by absorbing energy from the surroundings, or rearrange themselves, bond with other like-minded constituents, get ready for further life journey and are born. The cycle is repeated infinitely to continuously refresh and renew each and every part of the Universe. In short, we may call it the Law of Reproduction. Since, each and every smallest constituent is unique in itself at each and every point of time; it will be reborn as a different being. We can conclude that the concept of rebirth exists in the Universe but the re-born product is never the same. It will always be somewhat different from its earlier form.

The Universe is continuously changing, rearranging and evolving itself. There could be innumerable number of visible and invisible species living in various parts of the Universe, all of them passing through the cyclical life spans and doing their bit for maintaining the dynamic equilibrium.
Constituents with temporarily shorter life spans appear alive to us while those with relatively longer ones appear inert, though all have life and are made up of live smaller constituents.

\section{Conclusion}

The God is considered as omnipresent, round the clock overseeing the activities going on in the Universe. He even knows what is going on inside the minds of His creations. It is He, who orders and controls the activities. He rearranges the constituents to balance the forces in operation at a particular point of time. The forces may be 1 . the physical i.e. gravitational, electromagnetic, weak and strong; 2 . the nonphysical i.e. the 'soul force', the 'desire force', and the ' love force' and 3. 'the need force'. The need force of the constituents is the one on the basis of which the God drives its constituents to live through their lives. It may be defined as the force exerted by a constituent on the Universe which is needed for its most efficient working.

The physical forces are measurable external forces which make impact on the constituents at the macro level while the non-physical and the need forces are internal non-measurable forces and are individualistic in nature.

The non-physical forces may appear to some as the non conventional ones but everybody knows in the core of his heart regarding their existence, their power and effect as well. It is through these forces that a constituent makes efforts, creates need and send force waves in the Universe to get it deviated from Its normal chartered path. The Universe then rearranges Itself for fulfilling the need of the constituent in the best possible way.

Just as the physical forces consist of force particles, the non physical and the need forces also emit such particles, each individual particle being mass less and unique in itself. We may christen a soul force particle as 'Imhon'; a desire force particle as 'Lubon'; a love force particle as 'Priyon' and a need force particle as 'Unon'.

Depending upon the relative intensity of the physical, non-physical and the need forces the Universe continuously rearranges itself so that a dynamic equilibrium is always maintained. In short, whatever is needed by a constituent for the most efficient functioning of the Universe is being provided by the Universe to it at the most suitable point of time. Meaning thereby that the Universe is a self driving system with each of Its move being Unique in itself. We can summarize that the life in all the constituents of the Universe in its collective form represents something very great and supernatural. This is exactly what one perceives the image of the God.

The aforesaid discussion indicates towards only one and the unique solution- the God is Universe and the Universe is God. Both are exactly one and the same. Only the names are different. Once it has been proved beyond any reasonable doubt that both the names represent one and the same thing, we can coin a joint name. Hence, the title of this paper: 'Almighty, the Universe'. 
It is the entirety of the Universe including the mass, energy, forces and the collective life in Its constituents which we perceive as the God Almighty. He is indivisible, uncuttable, un-replicable, un-replaceable, un-createable, un-destroyable and unique. He is only one, whom we address by different names in different parts of our very small planet. He is the Creator of Himself embodying the life in the collective form who by Himself sitting on the driver's seat manages His affairs in the best possible and the most efficient manner.

Everybody has seen or felt the majesty of the Universe but only partly i.e. Its very tiny part only. Nobody has seen or felt It completely and nobody will, as it is not practically possible for anybody to see or feel the entire Universe. One should always remember that the spread of Its dimensions is not finite. However, one can always wander his imagination after closing his eyes and with focused concentration anywhere, everywhere, throughout and inside the Universe. Then he will be able to appreciate the grandeur of Its majesty as also Its competency in creating the constituents so skillfully and flawlessly. After-all It is 'Almighty, the Universe'.

\section{References}

[1] Ptolemy.(1515). "Almagest"

[2] Copernicus.(1536). "Commentariolous"

[3] Albert Einstein.(1916). "Theory of Relativity"

[4] Heisenberg.(1927). "Uncertainty Principles"

[5] Edwin Hubble.(1929). "The Hubble's Law"

[6] Brian Greene.(1999). “The Elegant Universe"

[7] Stephen Hawking.(2006). "Theory of Everything"

[8] Aristotle. 'Classical Element'

[9] Galileo.(1610).“The Starry Messenger”, (1655) "The Little Balance", "On Motion, (1602) "Mechanics"

[10] Newton.(1687).“Philosophiae Naturalis Principia Mathematica"

[11] Charles Darwin.(1859). "On the origin of Species"

[12] Georges Lemaitre.(1927). "Big Bang Theory"

[13] Wikipedia

[14] Divine Intervention 